\title{
A protocol for accurate radiochromic film dosimetry using Radiochromic.com
}

\author{
Ignasi Méndez¹, Juan José Rovira-Escutia², Bozidar Casar ${ }^{1}$ \\ ${ }^{1}$ Department for dosimetry and quality of radiological procedures, Institute of Oncology Ljubljana,Ljubljana, Slovenia \\ ${ }^{2}$ Centro Nacional de Dosimetría, INGESA, Valencia, Spain
}

Radiol Oncol 2021; 55(3): 369-378.

Received 24 June 2021

Accepted 01 July 2021

Correspondence to: Ignasi Mendez, Ph.D., Department for dosimetry and quality of radiological procedures, Institute of Oncology Ljubljana, Zaloška 2, SI-1000 Ljubljana, Slovenia. E-mail: nmendez@onko-i.si

Disclosure: Ignasi Méndez and Juan José Rovira-Escutia are co-founders of Radiochromic.com.

This is an open access article under the CC BY-NC-ND license (http://creativecommons.org/licenses/by-nc-nd/4.0/).

Background. Radiochromic films have many applications in radiology and radiation therapy. Generally, the dosimetry system for radiochromic film dosimetry is composed of radiochromic films, flatbed scanner, and film analysis software. The purpose of this work is to present the effectiveness of a protocol for accurate radiochromic film dosimetry using Radiochromic.com as software for film analysis.

Materials and methods. Procedures for image acquisition, lot calibration, and dose calculation are explained and analyzed. Radiochromic.com enables state-of-the-art models and corrections for radiochromic film dosimetry, such as the Multigaussian model for multichannel film dosimetry, and lateral, inter-scan, and re-calibration corrections of the response.

Results. The protocol presented here provides accurate dose results by mitigating the sources of uncertainty that affect radiochromic film dosimetry.

Conclusions. Appropriate procedures for film and scanner handling in combination with Radiochromic.com as software for film analysis make easy and accurate radiochromic film dosimetry feasible.

Key words: radiochromic film; dosimetry; protocol, film analysis software

\section{Introduction}

Radiochromic films are extensively employed in radiology and radiation therapy because they have excellent spatial resolution, near water-

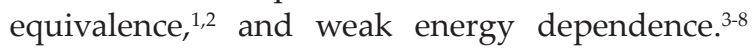
Furthermore, they can be immersed in water, ${ }^{9}$ can be cut, do not need chemical processing, and present low sensitivity to visible light. The active component of radiochromic films are diacetylene monomers which polymerize upon irradiation. ${ }^{10}$ Polymerization makes films increasingly dark with the absorbed dose. Changes in the visible absorption spectrum can be measured with a flatbed scanner. Scan pixel values are converted into doses with software for radiochromic film analysis. Hence, the dosimetry system for radiochromic film dosimetry commonly consists of radiochromic films, flatbed scanner, and film analysis software.

The purpose of this work is to present a protocol for accurate radiochromic film dosimetry using Radiochromic.com (Radiochromic SL, Benifaió, Spain) as software for film analysis.

Radiochromic.com is a software as a service (SaaS) program for radiochromic film dosimetry, radiotherapy QA, and image analysis. It aims to implement state-of-the-art methods and corrections for radiochromic film dosimetry. At the time of writing this work, Radiochromic.com was at version 3.3 . 


\section{Materials and methods}

The film-scanner system

\section{Radiochromic films}

This protocol is designed for the analysis of GafChromic films (Ashland Inc., Bridgewater, NJ, USA). In radiation therapy, GafChromic EBT3 and EBT-XD films are recommended. The difference between EBT3 and EBT-XD films lies in the length of the needles of the active component. EBT-XD needles are shorter, which leads to less darkening for the same absorbed dose and to higher saturation doses. Therefore, EBT-XD films are recommended for higher doses. EBT3 films can be used for applications with doses in the range of 0.01-20 Gy. However, for doses larger than $10 \mathrm{~Gy}$ and up to $40 \mathrm{~Gy}$, EBT-XD films are preferred. ${ }^{10}$

EBT3 and EBT-XD films are considered energy independent for MV photon beams. However, they under-respond to photon energies lower than 100 $\mathrm{keV}^{8}$, and exhibit LET dependence for protons. ${ }^{10}$

In $\mathrm{kV}$ X-rays applications, such as dose measurements in interventional radiology or IORT ${ }^{11}$, XR-RV3 films should be used instead. In the energy range of these applications, films are strongly energy dependent and should be calibrated for each energy in use. ${ }^{12}$

\section{Flatbed scanner}

Epson Expression 10000-12000XL and Epson Perfection V700-850 flatbed scanner models (Seiko Epson Corporation, Nagano, Japan) are recommended. They have been extensively studied in the literature and possess suitable characteristics for film dosimetry, such as RGB color channels, 16 bit color depth per channel, resolution up to 4800 dpi, reflection and transmission scanning modes, and lamp autocalibration. Epson Expression 1000012000XL have A3 size, while Epson Perfection V700-850 scanners have A4 size. Epson Expression 10000-12000XL are favored because they are less affected by the lateral artifact. ${ }^{13-20}$ Epson scanners can be controlled with the associated Epson Scan software or with alternatives such as VueScan (Hamrick Software, Phoenix, AZ, USA).

Papaconstadopoulos et al. ${ }^{21}$ found that the slope of the sensitometric curve in reflection scanning was flatter than in transmission mode and considered that the dose range should be reduced accordingly to doses lower than 2 Gy (red channel) and 8 Gy (green channel) in reflection mode. This assumption was called into question by Ramos and
Pérez Azorín ${ }^{22}$ who argued that the dose range should not be defined in terms of absolute changes of the signal with the dose but in relative terms by taking into consideration the signal to noise ratio. The protocol presented here is valid for both scanning modes.

\section{Sources of uncertainty}

Several sources of uncertainty contribute to the total uncertainty of the film-scanner system. Some of them affect radiochromic films, others are exclusive of the scanner, while the rest arise from the interaction between film and scanner.

\section{Uncertainties of radiochromic films}

Films display variations in the thickness of the active layer ${ }^{23,24}$, causing film heterogeneities and differences between films of the same lot (i.e., intra-lot variations). Also, film darkening continues indefinitely following irradiation, although at an ever slower pace. ${ }^{25}$ Humidity and temperature alter film response, yet this influence is reversible as long as the temperature does not reach more than $60^{\circ} \mathrm{C} .9,10,26,27$ Active layer polymerization can be noncatalytic or can also be induced by ultraviolet light. ${ }^{26}$ Finally, dust, scratches, and marks modify the response of the system.

\section{Uncertainties of the scanner}

Despite autocalibrating before each scan, scanners experience inter-scan variations, which cause that repeated scans do not deliver constant responses. ${ }^{15,18}$ The signal of the scanner have noise..$^{14,28,29}$ The scanner lamp should warm-up before use. ${ }^{30,31}$ And other minor sources of uncertainty of the scanner include grid patterns and positional inaccuracies. ${ }^{15}$

\section{Uncertainties of the interaction between film and scanner}

For a given dose value, the response of the filmscanner system in pixel value decreases with the distance to the center of the scan on the axis parallel to the lamp. Furthermore, this lateral response artifact becomes more important for higher doses. ${ }^{16,19,32}$ Radiochromic films polarize light, which means that the response of the system depends on the orientation of the film on the scanner bed. ${ }^{33}$ Also, the response depends on film-to-light source distance. ${ }^{18,34}$ The effect of the point spread function of the film-scanner system is usually negligi- 
ble, however, in high contrast regions may become significant. ${ }^{16}$ Lastly, even though the addition of microscopic silica particles to the film surface prevents the occurrence of Newton rings ${ }^{35}$, they are still a relevant source of uncertainty for older film models (i.e., EBT2).

\section{Image acquisition}

A protocol for accurate radiochromic film dosimetry must minimize the impact of all those sources of uncertainty. To this end, and regarding image acquisition, our protocol recommends:

1. Keep films in a dry and dark environment.

2. Handle films with care, do not touch them without wearing gloves to prevent marks and scratches.

3. Keep films away from light whenever possible.

4. If films are submerged in water, minimize the time of submersion.

5. Do not bend films when cutting them. Use sharp scissors or, preferably, a guillotine.

6. Films, either entire films or film fragments, shall always keep the same orientation (i.e., portrait or landscape) on the scanner. Label them to keep the orientation with the original sheet and place them consistently on the scanner.

7. Scanning the films prior to and after irradiation delivers more accurate results. ${ }^{23}$ However, it compels the use of a frame to place the films at the same position in both occasions and, according to our experience, reduces the uncertainty of film doses in less than $0.5 \%$. Consequently, scanning the films prior to irradiation is optional in this protocol.

8. After irradiation, wait for polymerization to stabilize. For convenience, films are usually scanned $24 \mathrm{~h}$ after irradiation. Short waiting times can be employed as well, however, in this case, time windows must be narrower to avoid losing accuracy. For instance, Devic et al. ${ }^{36}$ found similar errors with waiting-time windows of 24 $\pm 2 \mathrm{~h}$ and $30 \pm 5 \mathrm{~min}$. Different waiting-time windows are associated with different sensitometric curves. Even though these differences can be reduced with re-calibration methods ${ }^{37}$, this protocol recommends using the same waitingtime window employed during the calibration to prevent avoidable uncertainties.

9. Warm up the scanner for at least $30 \mathrm{~min}$ before use.

10. Before acquisitions, and after pauses, perform several (e.g., five) empty scans to stabilize the scanner lamp.

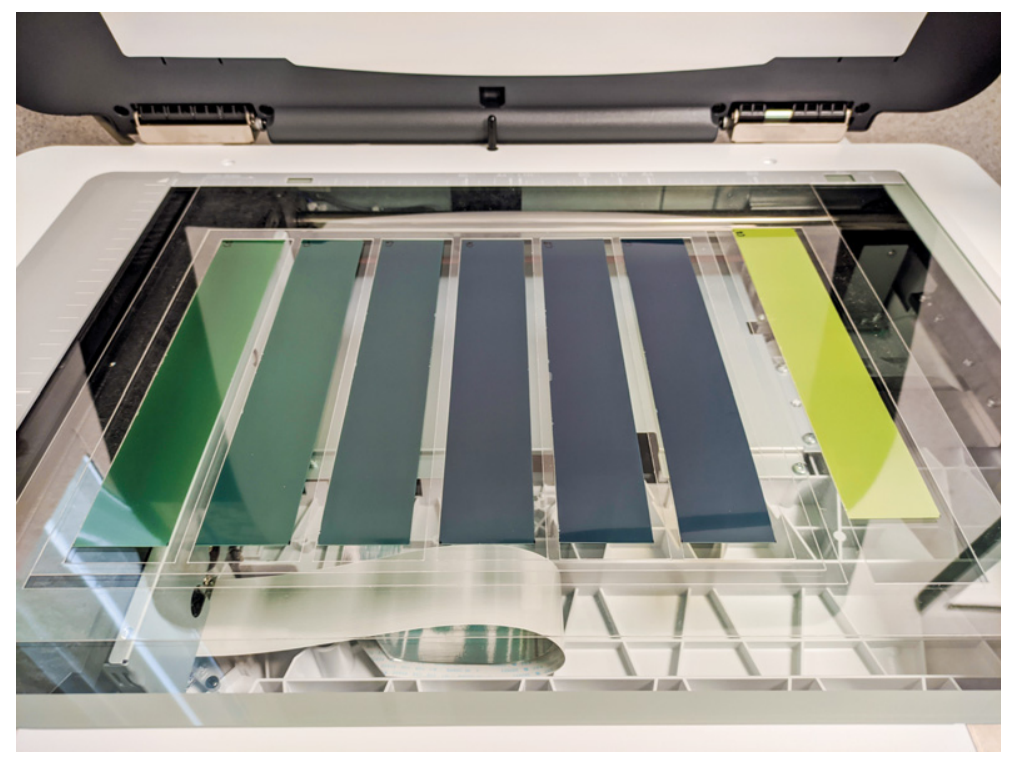

FIGURE 1. Positioning films on the scanner with a frame.

11. Center the film on the scanner. A convenient way to do so is with a frame. To prevent high contrast between frame and film, frames can be built from transparent materials, such as acetate transparencies or PMMA sheets. A set of technical drafts for frames and compression sheets suitable for the scanners recommended in this protocol can be found in the supplementary materials (Figures S1-S8). Figure 1 shows an example on how films can be positioned on the scanner with a frame.

12. Films shall be in perfect contact with the surface of the scanner bed to avoid curling. In transmission mode, place a $2-4 \mathrm{~mm}$ thick glass or PMMA sheet on top of the film. The positioning of the compression sheet shall be consistent, therefore, either cover or keep free the autocalibration area for all the scans. In reflection mode, the scanner lid itself compresses the film adequately.

13. Always use the same scanning mode, either reflection or transmission, that was used for the calibration.

14. Maintain a fix scanning area by saving it into the scanning software settings. In this manner, pixel positions on the film match with scanner coordinates, which is imperative when applying lateral corrections or scanning before and after irradiation.

15. Scan with 48 bit RGB mode and all image processing tools turned off.

16. A resolution of 50-75 dpi $(0.51-0.35 \mathrm{~mm})$ fits most applications. While for treatments using 


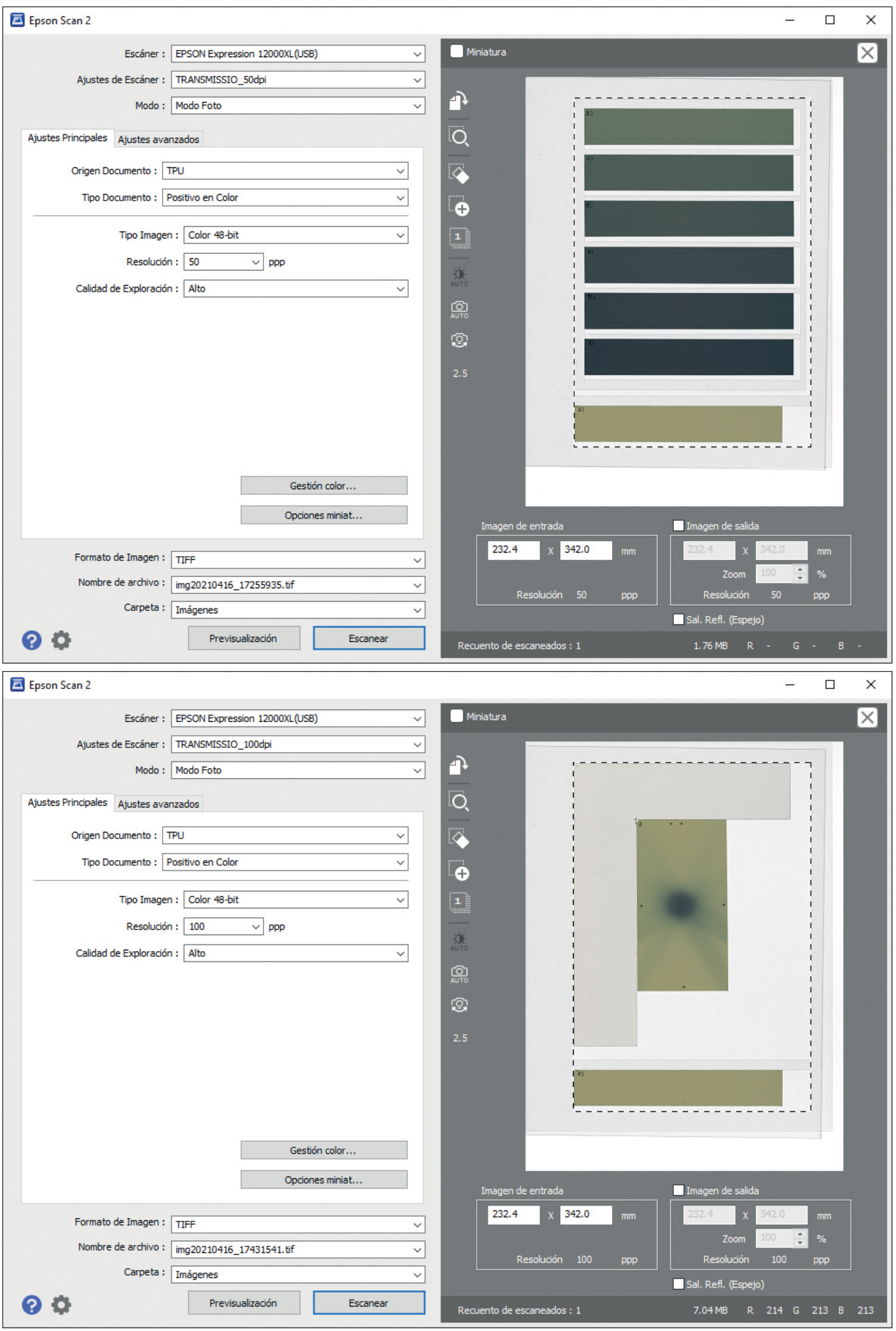

FIGURE 2. Scanner settings for (A) a calibration and (B) a treatment using small fields. 


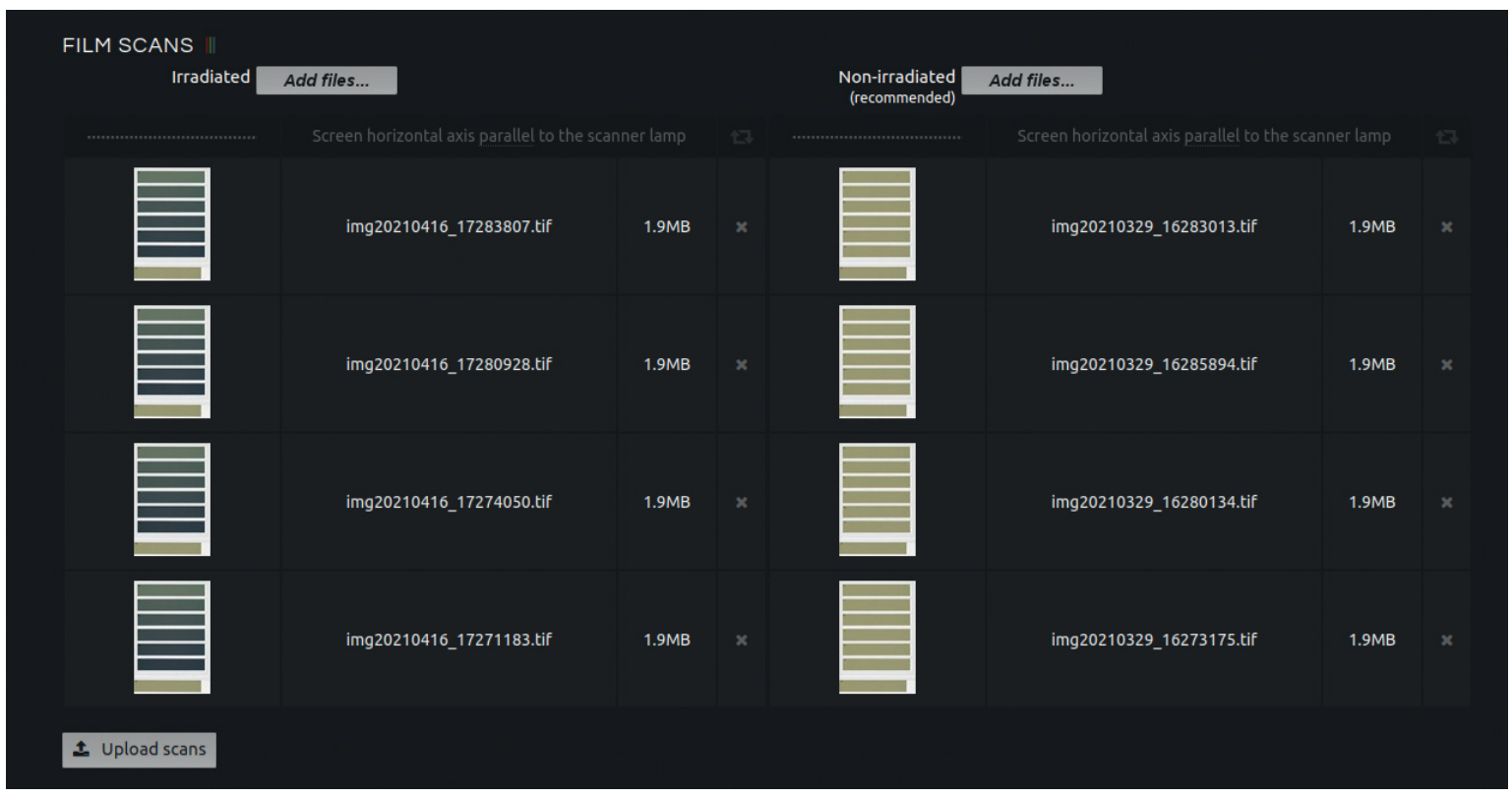

FIGURE 3. Upload films to Radiochromic.com with the correct orientation.

small fields 100-150 dpi (0.25-0.17 mm) may be necessary. In this protocol, higher resolutions are discouraged because they produce larger noise ${ }^{14,15}$ and slow down film scanning and analysis. Figure 2 depicts scanner settings for a calibration and a treatment using small fields.

17. Perform four or five repeated scans and discard the first one for each film.

18. Upload the scans to Radiochromic.com selecting the orientation of the scanner lamp on the scans. The orientation should be correct to apply lateral corrections. The software will acquire the average image of the film after irradiation (irradiated film), and optionally before irradiation (non-irradiated film). Figure 3 illustrates how films are uploaded to Radiochromic. com.

\section{Calibration and lateral correction}

\section{Calibration}

A calibration is needed to convert film images into absorbed dose distributions. On Radiochromic. com, film images are converted into doses following the Multigaussian model ${ }^{23}$ for multichannel radiochromic film dosimetry. The Multigaussian model considers that the probability density function of the film-scanner system's response given a dose $D$ follows a multivariate Gaussian distribution, where the response is a vector of pixel values including all color channels and both irradi- ated and non-irradiated films. Or, in mathematical form,

$P(\mathbf{z} \mid D) \sim \mathcal{N}(\boldsymbol{\mu}(D), \mathbf{\Sigma}(D))$

where $\mathbf{z}$ is the response vector, $\boldsymbol{\mu}$ is the expectation vector, and $\boldsymbol{\Sigma}$ is the covariance matrix.

On Radiochromic.com, a set of known reference doses are associated to regions of interest (ROIs) of a film image during the calibration. The outcome of the software consists of pairing each dose (D) with the median pixel values $(\boldsymbol{\mu}(D))$ and covariance matrix $(\boldsymbol{\Sigma}(D))$ of the response on the associated ROI. Radiochromic.com does not fit sensitometric curves, for dose values not included in the calibration, $\boldsymbol{\mu}(D)$ and $\boldsymbol{\Sigma}(D)$ are interpolated with natural cubic splines.

\section{Lateral response correction}

Commonly, to correct the lateral artifact, it is necessary to fit the parameters of a function that relates dose and distance to the center of the scan on the axis parallel to the lamp with changes in pixel values. Radiochromic.com integrates the calibration with the fitting of the lateral correction. To do so, the software applies the Lewis and Chan model for the lateral correction: ${ }^{17,38}$

$v(x)=\alpha_{1}\left(x-x_{c}\right)+\alpha_{2}\left(x-x_{c}\right)^{2}+$

$\hat{v}(x)\left(1+\beta_{1}\left(x-x_{c}\right)+\beta_{2}\left(x-x_{c}\right)^{2}\right)$

where $v$ is the pixel value after correction, $x$ is the coordinate on the axis parallel to the lamp, $x_{c}$ is 


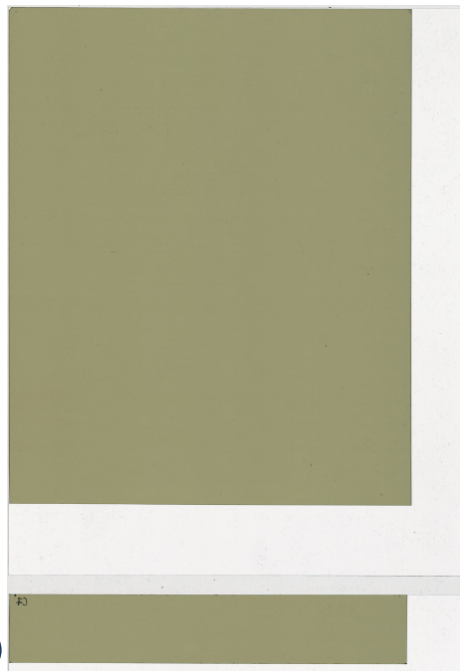

(B)

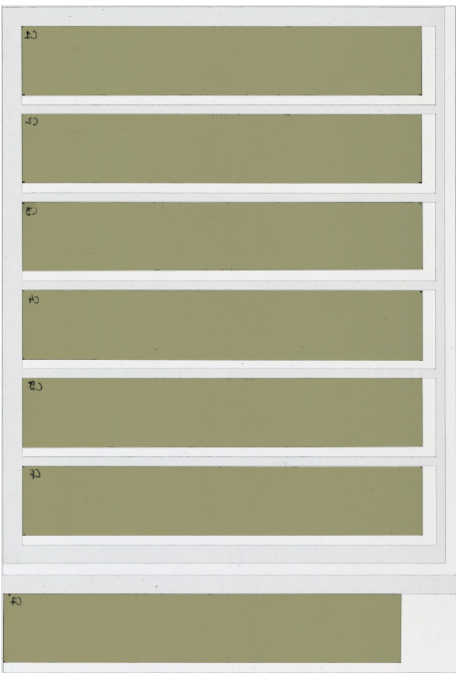

(C)

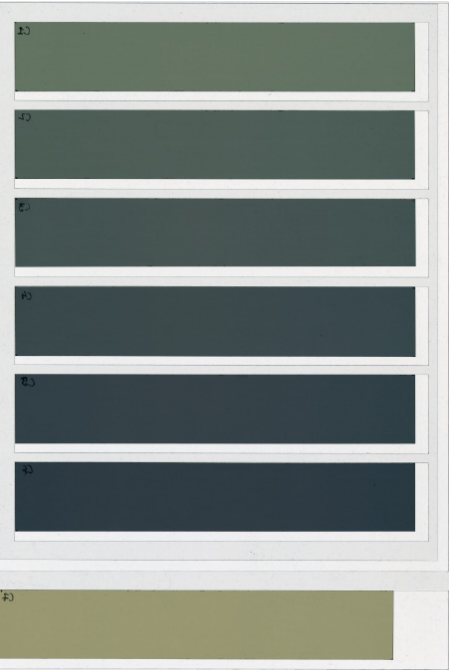

(B) calibration fragments prior to irradiation, and FIGURE 4. Scans for a calibration including
(C) calibration fragments after irradiation.

the coordinate of the center of the scanner, $\hat{v}$ is the pixel value before correction, and $\alpha_{1}, \alpha_{2}, \beta_{1}$, and $\beta_{2}$ are fitting parameters. As explained by Méndez $e t$ $a l^{23}$, scanning an unexposed film can simplify the process of fitting the parameters. We can rewrite the lateral correction formula as

$$
\begin{aligned}
& v(x)=v_{0}+\left(\hat{v}(x)-\widehat{v_{0}}(x)\right)\left(1+\beta_{1}\left(x-x_{c}\right)+\right. \\
& \left.\beta_{2}\left(x-x_{c}\right)^{2}\right)
\end{aligned}
$$

where $v_{0}$ is the pixel value at zero $x$ and $\widehat{v_{0}}(x)$ is the pixel value before correction as a function of $x$, both of them on the unexposed film. Furthermore, if the dose is homogeneous along the axis parallel to the lamp, equation (3) becomes

$\frac{v-v_{0}}{\hat{v}(x)-\widehat{v_{0}}(x)}=1+\beta_{1}\left(x-x_{c}\right)+\beta_{2}\left(x-x_{c}\right)^{2}$

Radiochromic.com follows equation (4). Thus, in order to calibrate and fit the lateral correction simultaneously, the reference doses should be homogeneous along the axis parallel to the lamp and the image of an unexposed film has to be uploaded to the application too.

\section{Procedure for the calibration}

In this protocol, we expose a calibration procedure for external photon beams, yet, other methods, radiation sources, and applications are possible, provided that they observe four basic principles:

Calibrations are valid for films from the same lot, therefore, each lot of films has to be calibrated at least once. However, since films slowly autopolymerize over time, it is advisable to repeat lot cali- brations from time to time. Furthermore, since film response depends on humidity and temperature, more accurate film doses can be expected when calibration and film dose measurements are done together.

Uncertainties in the absorbed reference doses will be translated into film dose uncertainties. Hence, it is important to maximize the accuracy of the reference doses. Generally, this can be achieved by irradiating at reference conditions and selecting ROIs with homogeneous doses.

To avoid the lateral response artifact, the ROIs with reference doses should be centered on the scan.

Finally, the reference doses should cover the range of doses of interest to prevent extrapolations.

In accordance with these principles, to calibrate photon beams from a linear accelerator we recommend:

1. If the calibration will include the lateral correction, acquire also the image of an unexposed film.

2. Cut a film into several (e.g., seven) strips with the longer side of the strips parallel to the lamp.

3. Keep one strip unexposed. One-by-one, irradiate the other strips at reference conditions in a water equivalent phantom. Strip doses should go from zero to a dose around 20\% larger than the largest dose of interest. If the calibration will include the lateral correction, irradiate the strips with approximately homogeneous doses by using a beam with flatenning filter and a $25 \mathrm{~cm} \times$ $25 \mathrm{~cm}$ field.

4. Scan all the calibration strips simultaneously. The irradiated areas of the strips should be centered on the scan. Figure 4 presents scans for a 


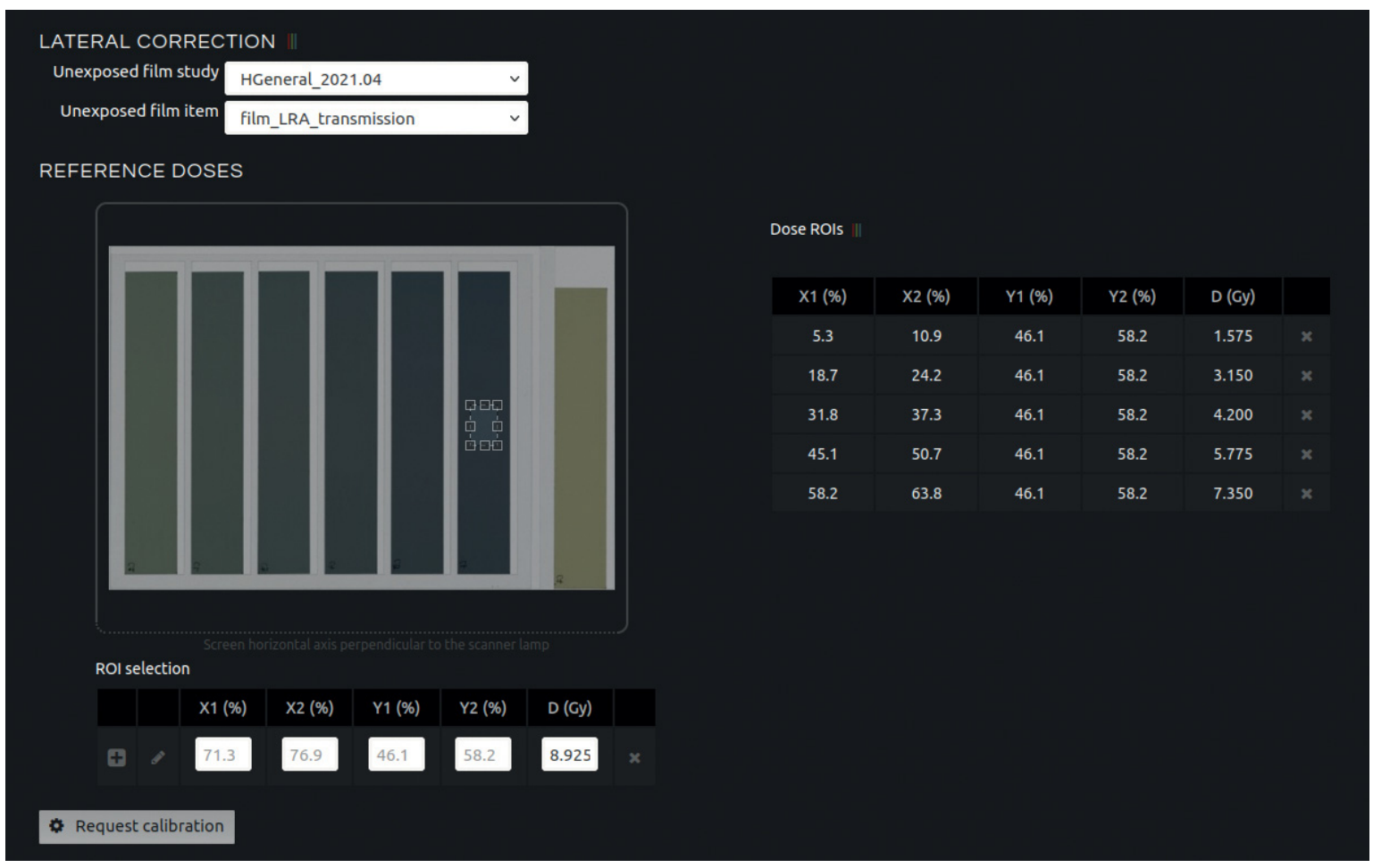

FIGURE 5. Associating reference doses to ROIs for a calibration which includes lateral correction.

calibration including lateral correction. An unexposed image and fragments irradiated with approximately homogeneous doses are necessary, the scan of the calibration fragments prior to irradiation is optional.

5. Keep the unexposed strip on the scanner and do not remove it until the next calibration. This will allow easier inter-scan corrections when measuring other films.

6. Acquire images into Radiochromic.com verifying that they are correctly oriented. For the calibration, doses should change on the left-right axis of the screen. Correct the orientation if they change on the vertical axis.

7. On the Calibration functionality of Radiochromic.com, select the calibration image. Select also the unexposed film image if the calibration will include lateral correction.

8. Associate reference doses to ROIs. The ROIs should be centered on the image (and on the scan). To provide enough statistics for the calibration while avoiding the lateral artifact, the length of the ROIs on the axis parallel to the scan should be between 1-4 cm approximately. An example of this process can be found in Figure 5 .

9. Radiochromic.com provides the mean error of the calibration, which computes the difference between film doses after applying the calibration to the pixels of the ROIs and reference doses. In our experience, calibrations have mean errors around $1-2.5 \%$. Larger errors may point to flaws in the procedure. Also, they can be expected for low doses, since uncertainties in radiochromic film dosimetry grow fast for doses lower than 1.5 Gy. ${ }^{39}$ To reduce uncertainties when measuring low doses with radiochromic films, we recommend to scale the number of MUs. For instance, when measuring MLC transmission with films, we recommend to irradiate with thousands of MUs to achieve film doses around 2 Gy.

\section{Dose calculation}

Once a calibration is made, the procedure to convert film pixel values into doses consists of:

1. Scan the film together with the unexposed strip of the calibration. Figure 6 shows an example of film scans before and after irradiation. A piece of transparency sheet was used, in addition to a frame, to position the film consistently on the scanner. However, scanning before irradiation is optional in this protocol.

2. Acquire the film image into Radiochromic.com and verify the orientation.

3. On the Dosimetry functionality of Radiochromic. com, select the film image and the calibration. 

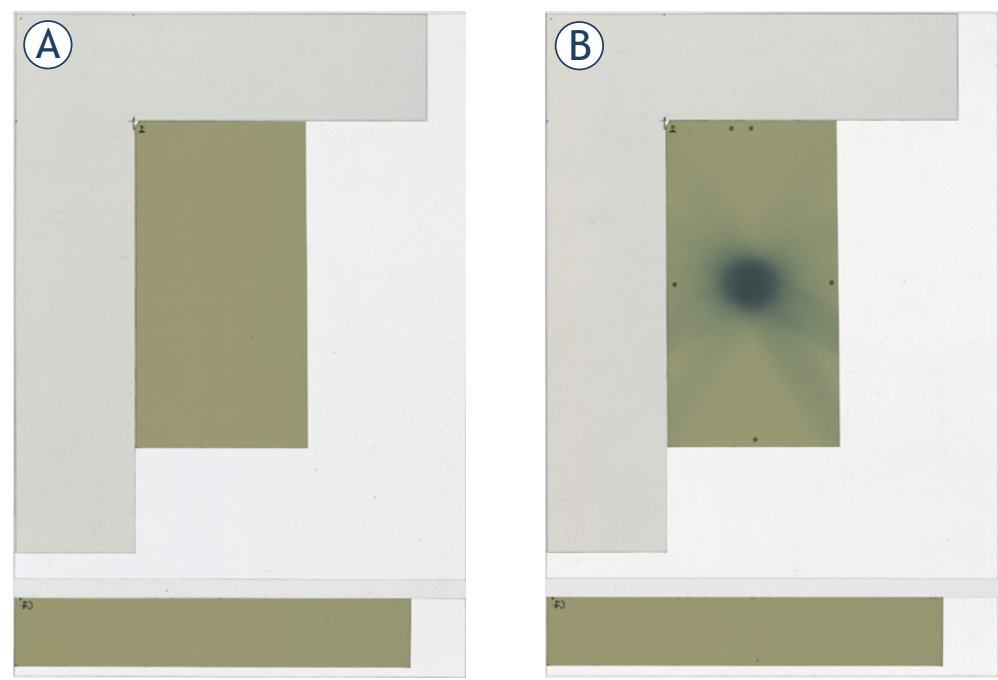

FIGURE 6. Scans of a film (A) before and (B) after irradiation. A piece of transparency sheet was used, in addition to a frame, to position the film consistently on the scanner. ed channels, both the calibration and the film to measure should be scanned before and after irradiation. Otherwise, only the irradiated channels are evaluated.

4. Keep the default noise reduction, which applies a $3 \times 3$ square median filter to the dose distribution.

5. Apply the inter-scan correction. To do so, select a ROI on the unexposed strip. The ROI should be centered on the scan to avoid the lateral artifact. For each color channel, the inter-scan correction multiplies each pixel of the image with a factor such that the median pixel value in the ROI coincides with the median pixel value of the unexposed ROI during the calibration. Figure 7 illustrates this process.

6. Re-calibration correction is optional in this protocol. In Radiochromic.com it is composed of inter-scan correction and dose rescaling. To apply dose rescaling, before the irradiation, cut a strip from the film to measure. This strip should be irradiated with a known homogeneous dose and scanned together with the rest of the film and the unexposed strip. Finally, select a ROI of the exposed strip centered on the scan and introduce its dose. Radiochromic.com will rescale film doses in order to match the median dose of the ROI.

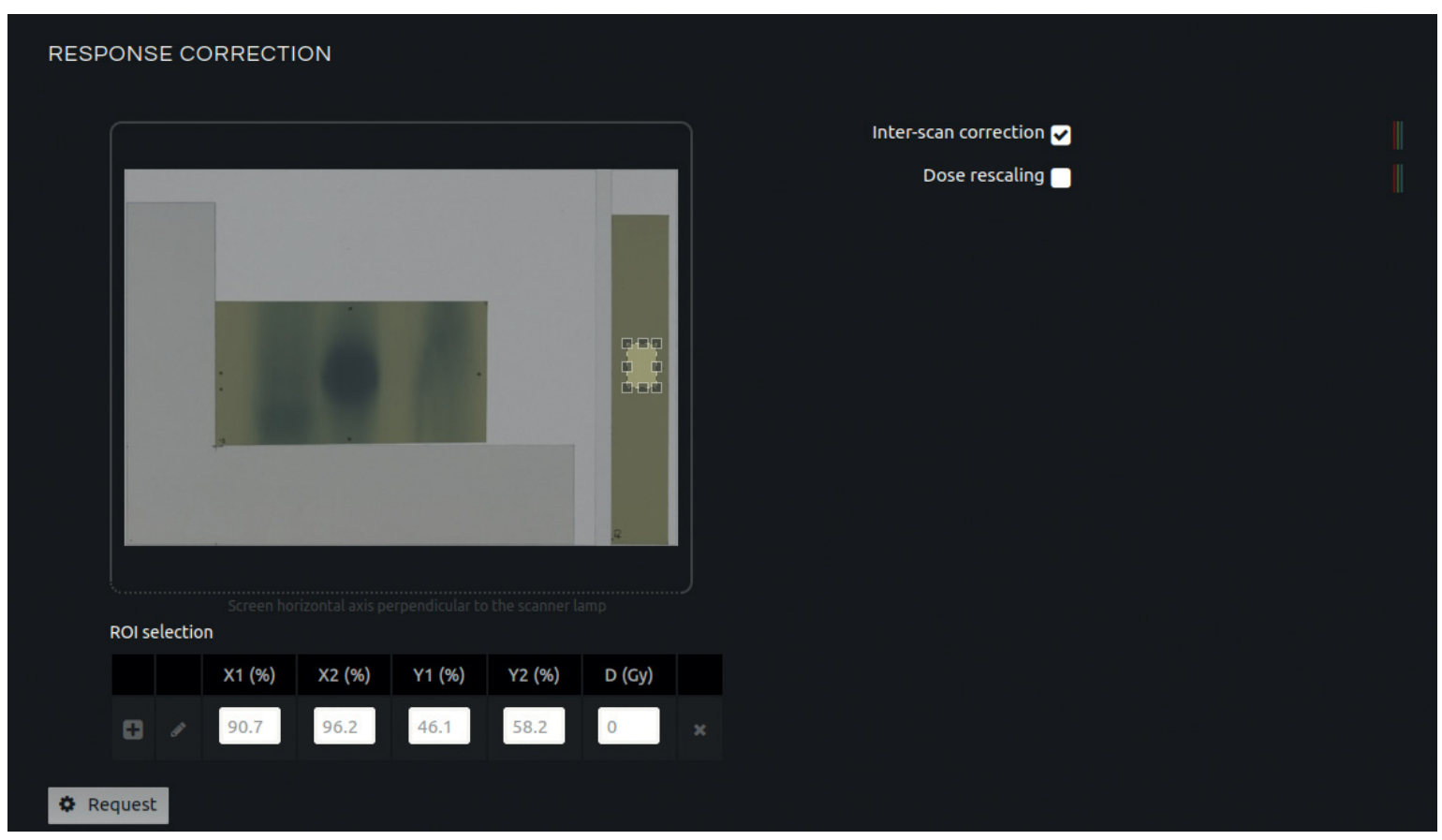

FIGURE 7. Applying the inter-scan correction in the film dosimetry functionality of Radiochromic.com. 


\section{Results and discussion}

The purpose of this work is to propose an accurate protocol that minimizes the sources of uncertainty that affect radiochromic film dosimetry by implementing appropriate procedures and up-to-date models and corrections. The protocol presented here incorporates the Multigaussian model for multichannel film dosimetry, and lateral, interscan, and re-calibration corrections of the response. Procedures for image acquisition, lot calibration, and dose calculation are stated in detail. The accuracy of this protocol has been validated repeatedly in several published studies. ${ }^{23,40,41}$

\section{Uncertainties of radiochromic films}

With respect to the way uncertainties are reduced, variations in the thickness of the active layer are mitigated in this protocol with the Multigaussian model, and to a larger extent if films are scanned before and after irradiation. The evolution of film darkening with post-irradiation time can be managed with a constant waiting-time window post-irradiation. Alternatively, the re-calibration correction can be used, which also mitigates humidity and temperature changes, intra-lot variations, and noncatalytic or ultraviolet-catalyzed polymerization. Dust, scratches, and other marks require a repetition of the measurements. However, repeated measurements with different films also reduce uncertainties due to film heterogeneities, intra-lot variations, and noncatalytic or ultraviolet-catalyzed polymerizations. Therefore, repeating measurements with different films is endorsed when the highest level of accuracy is needed.

\section{Uncertainties of the scanner}

The inter-scan correction mitigates inter-scan variations, which are also reduced by taking repeated scans of the same film. The noise of the scanner signal is reduced by taking repeated scans, by applying a square median filter to the dose distribution, and by the Multigaussian model. By repeting scans, minor positional inaccuracies of the scanner are averaged as well. Multiple measurements with different films reduce scanner noise, inter-scan variations, grid patterns and positional inaccuracies.

\section{Uncertainties of the interaction between film and scanner}

The lateral artifact is mitigated by the lateral correction. The dependency on the orientation of the film on the scanner bed is removed if films always keep the same orientation. And, by scanning in reflection mode or employing a compression sheet if scanning in transmission mode, dependency on film-to-light source distance can be reduced as well.

\section{Conclusions}

This work presents a protocol for accurate radiochromic film dosimetry using Radiochromic.com as software for film analysis. Detailed procedures for image acquisition, lot calibration, and dose calculation are explained and analyzed. State-of-theart models and corrections for film dosimetry, such as the Multigaussian model, and lateral, inter-scan, and re-calibration corrections of the response, are applied. Proper procedures for film and scanner handling in combination with Radiochromic.com software provide the means for easy and accurate radiochromic film dosimetry.

\section{References}

1. Crijns W, Maes F, van der Heide UA, V den Heuvel F. Calibrating page sized Gafchromic EBT3 films. Med Phys 2013; 40: 012102. doi: 10.1118/1.4771960

2. Niroomand-Rad A, Blackwell CR, Coursey BM, Gall KP, Galvin JM, McLaughlin WL, et al. Radiochromic film dosimetry: Recommendations of AAPM Radiation Therapy Committee Task Group 55. Med Phys 1998; 25: 2093115. doi: $10.1118 / 1.598407$

3. Rink A, Vitkin IA, Jaffray DA. Energy dependence ( $75 \mathrm{kVp}$ to $18 \mathrm{MV}$ ) of radiochromic films assessed using a real-time optical dosimeter. Med Phys 2007; 34: 458-63. doi: 10.1118/1.2431425

4. Richter C, Pawelke J, Karsch L, Woithe J. Energy dependence of EBT-1 radiochromic film response for photon (10 kVp-15 MVp) and electron beams (6-18 MeV) readout by a flatbed scanner. Med Phys 2009; 36: 5506-14. doi: $10.1118 / 1.3253902$

5. Arjomandy B, Tailor R, Anand A, Sahoo N, Gillin M, Prado K, et al. Energy dependence and dose response of Gafchromic EBT2 film over a wide range of photon, electron, and proton beam energies. Med Phys 2010; 37: 1942-7. doi: $10.1118 / 1.3253902$

6. Lindsay $P$, Rink $A$, Ruschin M, Jaffray $D$. Investigation of energy dependence of EBT and EBT-2 Gafchromic film. Med Phys 2010; 37: 571-6. doi: $10.1118 / 1.3291622$

7. Massillon-JL G, Chiu-Tsao S, Domingo-Munoz I, Chan M. Energy Dependence of the new Gafchromic EBT3 film: dose response curves for $50 \mathrm{KV}, 6$ and 15 MV x-ray beams. Int J Radiat Oncol Biol Phys 2012; 1: 60-5. doi: 10.4236/ ijmpcero.2012.12008

8. Bekerat H, Devic S, DeBlois F, Singh K, Sarfehnia A, Seuntjens J, et al. Improving the energy response of external beam therapy (EBT) GafChromic dosimetry films at low energies ( $\leq 100 \mathrm{keV}$ ). Med Phys 2014; 41: 022101. doi: $10.1118 / 1.4860157$ 
9. León-Marroquín EY, Lárraga-Gutiérrez JM, Herrera-González JA, CamachoLópez MA, Villarreal Barajas JE, García-Garduño OA. Investigation of EBT3 radiochromic film's response to humidity. J Appl Clin Med Phys 2018; 19 283-90. doi: 10.1002/acm2.12337

10. Niroomand-Rad A, Chiu-Tsao ST, Grams MP, Lewis DF, Soares CG, Van Battum $\sqcup$, et al. Report of AAPM Task Group 235 radiochromic film dosimetry: an update to TG-55. Med Phys 2020; 47: 5986-6025. doi: 10.1002/ mp.14497

11. Lozares S, Font JA, Gandía A, Campos A, Flamarique S, Ibáñez R, et al. In vivo dosimetry in low-voltage IORT breast treatments with XR-RV3 radiochromic film. Phys Med 2021; 81: 173-81. doi: 10.1016/j.ejmp.2020.12.011

12. McCabe BP, Speidel MA, Pike TL, Van Lysel MS. Calibration of Gafchromic XR-RV3 radiochromic film for skin dose measurement using standardized $x$ ray spectra and a commercial flatbed scanner. Med Phys 2011; 38: 1919-30. doi: $10.1118 / 1.3560422$

13. Lárraga-Gutiérrez JM, García-Garduño OA, Treviño-Palacios C, HerreraGonzález JA. Evaluation of a led-based flatbed document scanner for radiochromic film dosimetry in transmission mode. Phys Med 2018; 47: 86-91. doi: 10.1016/j.ejmp.2018.02.010

14. González-López A, Vera-Sánchez JA, Ruiz-Morales C. The incidence of the different sources of noise on the uncertainty in radiochromic film dosimetry using single channel and multichannel methods. Phys Med Biol 2017; 62: N525-36. doi: 10.1088/1361-6560/aa8f74

15. Méndez I, Šljivić Ž, Hudej R, Jenko A, Casar B. Grid patterns, spatial inter-scan variations and scanning reading repeatability in radiochromic film dosimetry. Phys Med 2016; 32: 1072-81.

16. Battum $L$ van, Huizenga $H$, Verdaasdonk $R$, Heukelom $S$. How flatbed scanners upset accurate film dosimetry. Phys Med Biol 2015; 61: 625-49. doi: $10.1088 / 0031-9155 / 61 / 2 / 625$.

17. Lewis D, Chan MF. Correcting lateral response artifacts from flatbed scanners for radiochromic film dosimetry. Med Phys 2015; 42: 416-29.doi: 10.1118/1.4903758.

18. Lewis $D$, Devic $S$. Correcting scan-to-scan response variability for a radiochromic film-based reference dosimetry system. Med Phys 2015; 42: 5692-701. doi: 10.1118/1.4929563

19. Schoenfeld AA, Poppinga D, Harder D, Doerner KJ, Poppe B. The artefacts of radiochromic film dosimetry with flatbed scanners and their causation by light scattering from radiation-induced polymers. Phys Med Biol 2014; 59: 3575-97.doi: 10.1088/0031-9155/59/13/3575

20. Alnawaf H, Yu PK, Butson M. Comparison of Epson scanner quality fo radiochromic film evaluation. J Appl Clin Med Phys 2012; 13: 3957. doi: 10.1120/jacmp.v13i5.3957

21. Papaconstadopoulos P, Hegyi G, Seuntjens J, Devic S. A protocol for EBT3 radiochromic film dosimetry using reflection scanning. Med Phys 2014; 41: 122101. doi: 10.1118/1.4901308

22. Ramos L, Perez Azorin JF. Comment on "A protocol for EBT3 radiochromic film dosimetry using reflection dosimetry using scanning" [Med Phys 41(12), 122101 (6pp.) (2014)]. Med Phys 2015; 42: 2096-7. doi: 10.1118/1.4914853

23. Méndez I, Polšak A, Hudej R, Casar B. The multigaussian method: a new approach to mitigating spatial heterogeneities with multichannel radiochromic film dosimetry. Phys Med Biol 2018; 63: 175013.

24. Hartmann B, Martišíková M, Jäkel O. Homogeneity of Gafchromic EBT2 film Med Phys 2010; 37: 1753-6. doi: 10.1118/1.3368601

25. Andrés C, del Castillo A, Tortosa R, Alonso D, Barquero R. A comprehensive study of the Gafchromic EBT2 radiochromic film. A comparison with EBT. Med Phys 2010; 37: 6271-8. doi: 10.1118/1.3512792

26. Girard $F$, Bouchard $H$, Lacroix F. Reference dosimetry using radiochromic film. J Appl Clin Med Phys 2012; 13: 3994. doi: 10.1120/jacmp.v13i6.3994

27. Rink A, Lewis DF, Varma S, Vitkin IA, Jaffray DA. Temperature and hydration effects on absorbance spectra and radiation sensitivity of a radiochromic medium. Med Phys 2008; 35: 4545-55. doi: 10.1118/1.2975483

28. Bouchard H, Lacroix F, Beaudoin G, Carrier J-F, Kawrakow I. On the characterization and uncertainty analysis of radiochromic film dosimetry. Med Phys 2009; 36: 1931-46. doi: 10.1118/1.3121488

29. Van Hoof SJ, Granton PV, Landry G, Podesta M, Verhaegen F. Evaluation of a novel triple-channel radiochromic film analysis procedure using EBT2. Phys Med Biol 2012; 57: 4353-68. doi: 10.1088/0031-9155/57/13/4353
30. Paelinck L, De Neve W, De Wagter C. Precautions and strategies in using a commercial flatbed scanner for radiochromic film dosimetry. Phys Med Biol 2007; 52: 231-42. doi: 10.1088/0031-9155/52/1/015

31. Ferreira B, Lopes M, Capela M. Evaluation of an Epson flatbed scanner to read Gafchromic EBT films for radiation dosimetry. Phys Med Biol 2009; 54: 1073-85. doi: 10.1088/0031-9155/54/4/017

32. Schoenfeld AA, Wieker S, Harder D, Poppe B. The origin of the flatbed scanner artifacts in radiochromic film dosimetry-key experiments and theoretical descriptions. Phys Med Biol 2016; 61: 7704-24. doi: 10.1088/00319155/61/21/7704

33. Butson MJ, Cheung T, Yu P. Evaluation of the magnitude of EBT Gafchromic film polarization effects. Australas Phys Eng Sci Med 2009; 32: 21-5. doi: 10.1007/BF03178624

34. Palmer AL, Bradley DA, Nisbet A. Evaluation and mitigation of potential errors in radiochromic film dosimetry due to film curvature at scanning. J Appl Clin Med Phys 2015; 16: 5141. doi: 10.1120/jacmp.v16i2.5141

35. Dreindl R, Georg D, Stock M. Radiochromic film dosimetry: consideration on precision and accuracy for EBT2 and EBT3 type films. Z Med Phys 2014 24: 153-63. doi: 10.1016/j.zemedi.2013.08.002

36. Devic S, Aldelaijan S, Mohammed H, Tomic N, Liang LH, DeBlois F, et al Absorption spectra time evolution of EBT-2 model Gafchromic film. Med Phys 2010; 37: 2207-14. doi: 10.1118/1.3378675

37. Ruiz-Morales C, Vera-Sánchez JA, González-López A. On the re-calibration process in radiochromic film dosimetry. Phys Med 2017; 42: 67-75. doi: 10.1016/j.ejmp.2017.08.013

38. Méndez I, Hartman V, Hudej R, Strojnik A, Casar B. Gafchromic EBT2 film dosimetry in reflection mode with a novel plan-based calibration method. Med Phys 2013; 40: 011720. doi: 10.1118/1.4772075

39. Vera-Sánchez JA, Ruiz-Morales C, González-López A. Monte Carlo uncertainty analysis of dose estimates in radiochromic film dosimetry with single-channel and multichannel algorithms. Phys Med 2018; 47: 23-33. doi: 10.1016/j.ejmp.2018.02.006

40. Casar B, Gershkevitsh E, Mendez I, Jurković S, Huq MS. A novel method for the determination of field output factors and output correction factors for small static fields for six diodes and a microdiamond detector in megavoltage photon beams. Med Phys 2019; 46: 944-63. doi: 10.1002/mp.13318

41. Casar B, Gershkevitsh E, Mendez I, Jurković S, Saiful Huq M. Output correction factors for small static fields in megavoltage photon beams for seven ionization chambers in two orientations - perpendicular and parallel. Med Phys 2020; 47: 242-59. doi: 10.1002/mp.13894 